\title{
Entre la escucha y el escuchar: psicoanálisis, psicoterapia y pobreza urbana en Buenos Aires
}

| ${ }^{1}$ María E. Epele I

Resumen: Partiendo de los resultados de la etnografía que vengo desarrollando desde el año 2013 en Centros de Salud en un barrio del Área Metropolitana de Buenos Aires, el objetivo de este trabajo consiste en problematizar la escucha, es decir, los modos de escuchar en su diversidad que participan como tecnologías en dichos tratamientos centrados en la palabra y orientadas a sectores populares y poblaciones marginalizadas. A través de la articulación de las perspectivas que en Antropología abordan las psicoterapias y tecnologías psi en contextos de pobreza urbana por un lado, $\mathrm{y}$ aquellas que estudian los sentidos, por el otro, en este trabajo los modos de escuchar son analizados en términos de acciones corporales, perceptuales y expresivas. Finalmente, se describen y analizan diferentes modalidades de escucha psicológica (reductora, fragmentaria y en formación), a través del reconocimiento de tres procesos que atraviesan los modos de escuchar en las psicoterapias y que se corresponden con las tensiones producidas por los modos de gobierno de lo sensible, de la pobreza y de la marginación en contextos de pobreza urbana.

> Palabras-clave: psicoanálisis; psicoterapia; pobreza urbana; etnografía; Buenos Aires.
1 UBA/CONICET. II GG. Buenos

Aires, Argentina. Correo

electrónico: mariaepele33@

gmail.com

Recibido: 22/03/2015

Aprobado: 12/07/2015 
Las investigaciones sobre psicoterapias, psicoanálisis y tratamientos psicológicos en Argentina, han sido desarrolladas desde diversas perspectivas y disciplinas (VEZZETTI, 1985; PLOTKIN, 2003; DAMOUSI; PLOTKIN, 2009; VISACOVSKY, 2002). Privilegiando el psicoanálisis, la mayoría de estudios se han concentrado en las clases medias y elites, con algunas investigaciones en el sistema público de salud, aunque principalmente en los servicios de salud mental de hospitales públicos (VEZZETI, 1996; PLOTKIN, 2003; DAGFAL, 2009; VISACOVSKY, 2002; 2009; LAKOFF, 2005). Sin embargo, el examen de las psicoterapias, específicamente aquellas de genealogía psicoanalítica en Centros de Salud barriales, desde una aproximación etnográfica y a la luz de la vida cotidiana, ha sido escasamente desarrollado.

Partiendo de los resultados de la etnografía que vengo desarrollando desde el año 2013, el objetivo de este trabajo consiste en problematizar la escucha y los modos de escuchar entre los psicólogos que llevan a cabo tratamientos centrados en la palabra y orientados a sectores populares y poblaciones marginalizadas en un barrio del Área Metropolitana de Buenos Aires. En lugar de examinar las técnicas terapéuticas en relación exclusiva con las orientaciones psicoanalíticas y psicológicas, este trabajo describe y analiza las particularidades de las acciones que incluye la escucha y el escuchar entre psicólogos que desarrollan psicoterapias orientadas a estas poblaciones. Partiendo de ciertos desarrollos sobre los sentidos en general y el escuchar en particular en Antropología y Ciencias Sociales (MERLEAU-PONTY, 1986; STOLLER, 1989; BARTHES, 1986; VAN EDE, 2009; INGOLD, 2000), en este trabajo se describen y analizan ciertas tensiones entre las escuchas expertas y otros modos de escuchar en el curso de estas psicoterapias que, atravesadas por procesos de expertización, popularización y marginalización, participan y dan forma a sus desarrollos, temporalidades, posibles efectos y diferentes eficacias. La diversidad de modos de escuchar en el desarrollo de las diferentes acciones que incluyen estos tratamientos, es entendido como un dominio en el que confluyen y expresan diferentes registros de lo sensible, modos de decir y sentir, diversos saberes y modelos de inteligibilidad expertos, legos, populares y marginales. Debido a las rápidas transformaciones en las características de la pobreza urbana (SVAMPA, 2005), las cartografías que guían la escucha psicológica, traducen en categorías que combinan diversos saberes expertos y legos, las complejas realidades y experiencias sociales de malestar y sufrimiento (STOLKINER, 2013; EPELE, 2013). 
Finalmente, interrogar etnográficamente las técnicas de escucha en estos tratamientos, devuelve a las acciones de escuchar su diversidad, las relocaliza en las coordinadas de la vida ordinaria y barrial y hace visible los registros de lo sensible (decible, audible y visible) en los modos de gobierno de la pobreza (lógicas de poder, económicas, discursivas, etc.). Es decir, la escucha y los modos de escuchar no sólo están marcadas por las lógicas institucionales y reguladas por saberes y tecnologías expertas. También, una creciente variedad de modos de sentir participa y modifica los repertorios y relaciones entre lo audible y lo inteligible (los sonidos, ruidos, silencios, sentidos, sus intensidades, umbrales, ritmos y disonancias), modeladas por los procesos de gobernar la pobreza urbana toman forma en esta área geográfica (ROSE, 1998; FOUCAULT, 2009).

\section{El gobierno de lo sensible y las tecnologías psi}

Desde diferentes disciplinas, la problematización de los sentidos ha promovido la revisión de las perspectivas universalistas y esencialistas de lo sensible y los modelos analíticos y reduccionistas de abordaje. Cuestionando la dominancia del ojo y la visión en Occidente desde el iluminismo, diferentes autores han revisado la epistemología que jerarquiza y caracteriza los sentidos, los separa analíticamente, y los naturaliza en relación a la biología de los órganos (MERLEAU-PONTY, 1986; JAY, 1993; BARTHES, 1986). ${ }^{1}$

Desde la denominada Antropología de los sentidos, además, se han investigado temáticas que privilegian ya sea el oído, gusto o el tacto, y se han elaborado perspectivas que incluyen explícitamente la participación de ciertos sentidos en el desarrollo mismo de la investigación (VAN EDE, 2009; STOLLER, 1989). Sin embargo, los modelos constructivistas y representacionales dominantes en este campo de estudios han sido cuestionados, ya que reproducen, de forma invertida o refleja, la disección y jerarquía analítica tradicional de los sentidos (INGOLD, 2000).

Por un lado, en lugar de construir y representar la variación cultural y social en base a una biología y física universal, la devolución al sentir y a los sentidos su carácter de acciones y movimientos corporales, hace posible determinar los modos en que participan y se entrelazan tanto en los procesos perceptivos y corporales, como en los modos de habitar los territorios (INGOLD, 2000). Por otro lado, en lugar de naturalizar, se hace posible interrogar las políticas que producen y regulan 
los procesos de sentir, y los modos de gobierno que los domestican, legitiman y/o sancionan en determinadas poblaciones (ROSE, 1998, EPELE, 2007).

Focalizando en los modos del oír y escuchar, estas acciones se entrelazan y confunden con otras (ver, tocar, etc.) en los procesos de sentir, que incluyen en sus desarrollos emociones y sentidos o significados. Los procesos de sentir, a su vez, se corresponden con una suerte de gobierno de lo sensible, por el que se reparten, regulan y definen las características y registros de lo audible, decible, visible, y sus vínculos con lo inteligible, en los modos de habitar y vivir por parte de diferentes poblaciones en determinados territorios (FOUCAULT, 2009; INGOLD, 2000; EPELE, 2010). Estas particiones y reparticiones de lo sensible se corresponden, entonces, con fracturas que la desigualdad social, la segregación territorial, la composición poblacional, los patrones laborales y programas asistenciales, las relaciones de género, y los modos de padecer, enfermar y morir. Los regímenes de lo sensible, entonces, se corresponden en la materialidad de sus particularidades y distribuciones las lógicas de poder, las economías locales y morales que asume el capitalismo contemporáneo en determinadas regiones y territorios (SVAMPA, 2005).

Diversos estudios en Antropología han examinado las psicoterapias, psicoanálisis y tratamientos psi en poblaciones que viven en las periferias de diferentes regiones y países (BIEHL; GOOD; KLEINMAN, 2009; DUARTE, 1986; LOCK; NGUYEN, 2010). La investigación de las tecnologías psi, han cuestionado los modos en que los saberes y técnicas expertas transforman desigualdades, violencias, incertidumbres y opresiones en sufrimientos sociales en individuales, y lo traducen en categorías diagnósticas, en un amplio espectro de contextos sociales, políticos y económicos (conflictos, guerras, exilios, desplazamientos y opresión rutinaria vinculada a la pobreza y desigualdad). De acuerdo a estas perspectivas, dichas tecnologías psi domestican y legitiman ciertos modos de decir, escuchar y ver, producen y reproducen repertorios inteligibles de malestares y dolencias que, entonces, son susceptibles de ser abordados por las tecnologías disponibles.

Bajo la apariencia de una aparente pasividad, y dependencia exclusiva de la palabra hablada, escuchar es siempre una actividad relacional y en plural, es decir, los diferentes modos de escuchar son acciones y movimientos que incluyen a otros y a otras realidades. En sus diferentes versiones (expertas, legas, cotidianas, etc.), 
los modos de escuchar se entrelazan con otros modos de sentir en las experiencias

de la vida ordinaria (ej. ver al escuchar). Escuchar como repertorio de acciones, también incluye una apertura y entrega hacia los otros y otras realidades, que en su despliegue compromete, modifica y borra de forma continua los bordes y límites (del yo-otro, sujeto-mundo, real-irreal, etc.) (BARTHES, 1986; ROSE, 1998; FOUCAULT, 2009).

Focalizando en las perspectivas y experiencias de los psicólogos, el objetivo de este trabajo consiste en examinar las características de la escucha psicológica, como tecnología terapéutica experta de las psicoterapias orientadas a sectores populares en Centros barriales del sistema público de salud de la Región Metropolitana de Buenos Aires. La escucha, como versión en sustantivo del escuchar, refiere a la técnica experta en dichas psicoterapias, cuya genealogía psicoanalítica es marcada además por las denominaciones de los psicólogos locales con las que se las denomina: "tratar" o trabajar con la palabra", "tratamientos con la palabra". En este trabajo problematizo las tensiones entre las escuchas expertas y otros modos de escuchar que tienen lugar en los tratamientos centrados en la palabra como un dominio en el que confluyen y se expresan tanto diferentes registros de los sensible, modos de decir y sentir, como saberes expertos y diferentes modelos de inteligibilidad, cuyas características regulan los desarrollos, temporalidades, y posibles efectos y eficacias.

Debido a las rápidas transformaciones en las características de la pobreza urbana, las cartografías que guían la escucha psicológica, traducen en categorías que combinan lo experto, lego, general y local, el incremento de desigualdad social y segregación territorial, la precariedad de las viviendas, la expansión de economías ilegales, la diversificación de violencias y abusos, la consolidación de programas de ingreso y asistencia, la modificación de las redes de sociabilidad, etc. (EPELE, 2013). Por lo tanto, las tensiones que atraviesan las técnicas de escuchar en contextos de pobreza urbana, producen una diversificación tanto en los saberes expertos y legos, y en los modos de sentir y escuchar, en los vínculos entre lo decible, audible e inteligible, y también en las características de las experiencias, acciones e intervenciones corporales y emocionales que incluyen. A través de los procesos que denomino de expertización, popularización y marginalización, las tensiones entre la escucha y otros modos de escuchar no sólo dan forma a diferentes modalidades técnicas de escuchar (reductora, fragmentada 
y en formación). También, participan, regulan y modifican los regímenes de lo sensible, en este caso, de las relaciones entre lo audible y lo inteligible (los sonidos, ruidos, silencios, sentidos, etc.), sus intensidades, umbrales, ritmos y disonancias, involucrados en el gobierno de la pobreza y la marginación social.

\section{Etnografías, psicoterapias y marginalización}

Este trabajo es parte de un estudio etnográfico que vengo llevando a cabo sobre psicoterapias de orientación psicoanalítica en un barrio del Área Metropolitana de Buenos Aires, desde inicios del 2013. La investigación de las psicoterapias de orientación psicoanalítica, y dirigidas a poblaciones marginalizadas es un ámbito privilegiado para examinar los modos reales y posibles de articulación (integración, rechazo, tensión, expulsión, evitación, fricción, reproducción, etc.) entre las realidades sociales actuales en rápidas transformación y los abordajes terapéuticos ofrecidos por las instituciones estatales en los barrios populares.

Por un lado, el acelerado incremento de la desigualdad económica, social y de salud en las últimas décadas ha transformado tanto los mapas sociales, como el repertorio, distribución y características de los malestares y padecimientos en estas poblaciones (ORTIZ-HERNÁNDEZ; LÓPEZ MORENO; BORGES, 2007; FERNÁNDEZ, 2013). Los modos de gobierno de la pobreza en las últimas dos décadas han transformado sus características y sus escalas al ritmo del: deterioro acelerado de las condiciones materiales de vida, y los ciclos de recuperación y crisis, la extensión de los programas de asistencia y de ingreso, el montaje de economías extractivas en poblaciones marginalizadas (drogas, juego, etc.), el aumento del hacinamiento y problemas de vivienda, la diversificación de las violencias (locales, de género, etc.), las modificaciones en las características y dimensiones de redes de sociabilidad, y la emergencia de nuevos modos de padecer, sufrir y morir (EPELE, 2010). Por otro lado, las transformaciones de las tradiciones y las tecnologías terapéuticas psi en un sistema público de salud precarizado por las reformas neoliberales (privatización, tercerización, precariedad laboral, trabajo ad honorem, etc.) han modificado los modelos de la inteligibilidad, las técnicas terapéuticas y de intervención respecto de los malestares y dolencias (ESCUDERO, 2003; IRIART; WATZKIN, 2006; FERNÁNDEZ, 2013).

El trabajo de campo, específicamente las entrevistas y la observación participante fueron llevadas a cabo tanto en centros de salud como en diversos 
espacios barriales (comedores, viviendas, plazas, etc.). También, fue desarrollado

en Centros de Salud del área (en reuniones, ateneos, etc.). Además, fueron realizadas 25 entrevistas a profesionales que atienden en el barrio, y 30 entrevistas a pacientes (21 mujeres, 9 varones) mayores de 18 años y residentes del barrio. El estudio fue aprobado por el Comité de Ética, y se implementó el consentimiento informado a los participantes.

Como en otros servicios del sistema público de salud en la zona considerada, las modificaciones y deterioro constante durante las últimas décadas se evidencia en la recurrente o cíclica escasez de insumos, insuficiencia o inadecuación edilicia, precariedad de trabajo de los profesionales, que incluye el trabajo ad honorem (psicólogos en formación, recursos de formación y supervisión) como parte de las estructuras de atención. Las poblaciones, por otro lado, tienen una composición compleja. Además de los residentes locales, hay migrantes principalmente del interior del país y de otros países latinoamericanos. También, hay residentes que llegan de otros barrios de clase media y media alta, que se han mudado a este vecindario por motivos económicos.

\section{Sobre la escucha experta}

Desde los inicios del trabajo de campo sobre aquellas psicoterapias orientadas a poblaciones marginalizadas, la escucha y el escuchar como problemas terapéuticos, atravesaban los análisis de casos, espacios de formación, supervisiones grupales y debates de los psicólogos, entrevistas y conversaciones conmigo llevadas a cabo en el desarrollo de esta investigación. También, ciertos desarreglos del hablar y el escuchar, que referían a modos característicos que vienen estructurando la sociabilidad en estos barrios, participaban directa o indirectamente en el repertorio de los motivos de consulta entre aquellos residentes que se convierten en pacientes. Además de "no puedo dormir", o "estoy mal porque se murió mi hijo", se combina con "no tengo con quién hablar", "nadie te escucha", "tengo miedo de hablar", "hacen que te ponen la oreja, pero no escuchan", "no puedo andar hablando".

De expresión calma, ojos despiertos y hablar pausado, como parte de nuestro primer encuentro, Patricia me iba introduciendo los problemas más serios de la gente del barrio los que venía escuchado en diferentes en diferentes áreas del trabajo institucional. Guardando cierto formato de un saber experto, Patricia enumeraba, aquel repertorio de problemas, malestares y dolencias, expresado 
en una serie de categorías cuasi expertas, legas, locales y cotidianas (desempleo, maternidad adolescente, problemas de vivienda, violencias y abusos, conflictos económicos, consumo de diversas sustancias y adicciones en la familia, sufrimientos relacionados a muertes violentas de personas cercanas, ataques de pánico, insomnio, enfermedades psicosomáticas, problemas de aprendizaje, conflictos relacionados a actividades ilegales, entre los principales). En una suerte de lengua franca, estas categorías ya habían sido revisadas y apropiadas por las poblaciones no sólo que se atendían en el Centro de Salud, sino la mayoría de aquellos residentes que transitaban los centros y lugares de reunión en el barrio.

Para escuchar a otro, es práctica. Cómo te explico?...

Mirá... Rosario...es un caso que viene hace tiempo dando vueltas acá. Es de acá del barrio. Cuando me la deriva un compañero que se fue, venía con rótulos, "medio caño". Era un caso complicado... que venía con un "de riesgo". Con eso no se puede hacer mucho. . Es que Rosario tiene una historia increíble, es de libro, una novela...de terror.

En ese momento lo tomaba así... como decirte...lo oía, como a la distancia, y quedaba ahí. Seguía trabajando con lo más urgente. Porque tenía mucha cosa, con los hijos, la vivienda. Algunas se fueron despejando, solucionando, otras no. [...]

Empezó a contar lo que le había pasado, todo lo que le habían hecho, como había sobrevivido, era una historia increíble. Que para mí, al principio esto ya lo había contado a otros acá, pero era considerado como medio un delirio. Cosa que no lo es. Yo no me podía imaginar que alguien pudiera vivir eso. Igual lo tomaba... pero hasta ahí. Rosario no se lo contaba a nadie, lo hablaba acá. Desaparecía, un tiempo... después volvía... con mucha angustia, pesadillas.

Llegué... digamos, segura... por esto, una evaluación diagnóstica de que no era un delirio. Pero no poder creer que alguien haya pasado por tanta cosa ¿no?, como una cosa más de humanidad, de... como que no puede ser... Son como dos cosas distintas...no es que no le creo porque miente o delira, es que no se puede creer... Después de dos años... pudimos como volver a reconstruir... porque a mí me pasaba algo de que... se ve que... me costaba en ese momento, en ese momento me costaba mucho... creer, porque sé que es así... Pero creer en el sentido de no te puedo creer... ¿ ¿viste? Escucharla, Rosario, pudo ir hilvanando, su vida (Patricia, psicóloga).

Rosario era una entre ciertos pacientes que se habían convertido en casos paradigmáticos. No sólo Patricia, otros psicólogos, me habían hecho referencia al caso de Rosario, para señalarme ostensivamente como era trabajar en el Centro de Salud. De acuerdo a estos psicólogos, la escucha psicológica, y las acciones de escuchar en los tratamientos con estas poblaciones tienen un conjunto de características que las diferencian de otros que, centrados también en la palabra, son llevados en consultorios y centros privados de atención. 
En primer lugar, y como Patricia refiere, además del aprendizaje teóricotécnico, a escuchar se aprende escuchando, siendo escuchada y escuchando a otros (siendo analista, supervisando y en el propio análisis). ${ }^{2}$ Estas acciones de escuchar, no obstante están modeladas por ciertas dinámicas que regulan el proceso de atención en el sistema público de salud. Entre ellas encontramos: la no elección del terapeuta, la menor duración de la sesión, la fragilidad en la continuidad del sistema de atención (por huelgas, feriados, vacaciones, etc.), la falta de adecuación de los horarios de atención al régimen horario de trabajo, las listas de espera, las dificultades para aquellos que tienen planes sociales para regular los ritmos de tratamiento, y la menor duración de los tratamientos en el tiempo por la alta demanda. De este modo, las acciones de escuchar están sometidas a ciertos ritmos institucionales, que no sólo regulan sus tiempos (de duración de las sesiones, de los tratamientos, de sus interrupciones, y de las consultas de urgencias). También, están modeladas por la continuidad de los mismos u otros profesionales en el curso de los tratamientos (derivaciones, cambios, etc.). Estas restricciones, sin embargo, se complementan con una mayor amplitud y diversidad de recursos disponibles frente a diferentes tipos de urgencias (disponibilidad a través de teléfonos institucionales y personales, otros psicólogos disponibles a los que se puede recurrir, cercanía del Centro de salud respecto de la vivienda, etc.).

En segundo lugar, de acuerdo a los psicólogos, para poder escuchar a otro, hay que: "vaciar las palabras de sentido común", "despejar los prejuicios", resistirse a "saber de antemano quién es el otro", "evitar la tentación de haber entendido". Como dice Gabriela: "yo estoy escuchando que acá hay algo de que... que está sufriendo, de que la está pasando mal. Eso de adjudicarle el sentido común, no”. Esta condición de la escucha refiere a una ruptura entre los vínculos habituales entre los sonidos y sentidos, un extrañamiento respecto a lo dicho por los otros, y respecto a quienes son estos otros que viven otras realidades. Estas "rupturas", "saber de antemano", “despejar los prejuicios”, señalaban la búsqueda de la suspensión de la posibilidad de recurrir a modelos de inteligibilidad disponibles, en algo denominado como "sentido común".

En tercer lugar, en las conversaciones y entrevistas, los psicólogos diferenciaban diferentes tipos de acciones de escuchar en los tratamientos y que se hacen en el trabajo con estas poblaciones. Estas acciones son diferenciadas ya sea en términos de "oir " y "escuchar", entre escuchar "por arriba" y escuchar "realmente", 
entre "lo obvio y lo excepcional". Aunque binarias en su primera expresión, estas diferenciaciones distan mucho de serlo. En el detalle con el que Patricia me narraba la vida de Rosario, se combinaban una suerte de excepcionalidad y extrañeza respecto de ciertas experiencias que le había tocado atravesar, mientras que otras, ligadas a problemas familiares y de vivienda, ya formaban parte ya del repertorio conocido de la pobreza. Algo de lo que Rosario decía, si bien era oído por Patricia, era "tomado” y “dejado ahí”, era demasiado para incluir en el repertorio de "lo obvio" de los modos sociales de producción del sufrimiento al que los psicólogos ya estaban habituados en estos barrios. Este nivel de extrañeza que producían las experiencias de ciertos pacientes entre los psicólogos, los que en su mayoría eran de las clases medias, exceden al resguardo teórico/técnico de suspender el sentido común, necesario para que la escucha experta tenga lugar. En casos como el de Rosario, las acciones corporales, perceptuales y expresivas que involucran el escuchar en los tratamientos, van modificando las coordenadas habituales de lo sensible, señala las restricciones de la escucha experta y de las teorías dominantes que la modelan, e involucra temporalidades diferentes para darle estatuto de realidad, es decir, para su integración a lo audible, decible y visible en el contexto terapéutico.

En cuarto lugar, la mayoría de los psicólogos coinciden en otra condición, no basta con oír pasivamente, "hay que intervenir", ya sea con palabras, gestos y silencios. La intervención es "necesaria” para que el tratamiento continúe. De acuerdo a los propios psicólogos, su hablar es un modo de "alojar al otro", y a lo que le pasa al otro. ${ }^{3}$

Marcela sintetizaba

Cuando pasa la primera entrevista, si continúa o no, tiene que ver, con el momento del paciente. Las ganas que tenga de entregarse, de entregar lo que le pasa. De algún modo hay que entregarse. Y de a poco la posibilidad que tenés vos de alojar lo que le pasa a ese paciente, tu momento de la vida y alojar eso que te está contando.

Uno no dice mucho, en realidad son como cosas que vas... diciendo... poquito. Y tiene efecto, tiene como que... o sea, como que... como que es que alguien en el mundo me está escuchando, ¿viste?, alguien me está entendiendo. Eh... y por ahí viene... pero es como que uno también lo mide con el efecto que se tiene. Como que nada es calculado y nada es medido, las cosas que salen... no lo pensás a priori, como muy... Uno va escuchando y va... como... lanzando algunas cosas.

Además, se interviene con la mirada. A diferencia del psicoanálisis tradicional, con diván, el tratamiento centrado en la palabra en centros de salud 
en estas poblaciones, incluye la relación cara a cara. "Hay que saber trabajar

con la mirada. Podés evitar o poner cara de nada para que el otro pueda hablar, el semblante". También con la mirada se puede "sostener", "intervenir", "hacer gestos", "reforzar", "estar abierto".

En quinto lugar, el desarrollo de los tratamientos, y las acciones de escuchar y hablar en los mismos se ven modulados también por las condiciones edilicias, las características de los consultorios disponibles para el servicio de salud mental y el lugar de emplazamiento de los Centros de salud en los barrios. Además, la mayoría de los pacientes no sólo son atendidos por otros profesionales (médicos, enfermeros, etc.), sino que los más "vulnerables" son integrados a programas de intervención, es decir en sus viviendas y en articulación con otros programas e instituciones. Estas condiciones de atención en los Centros de Salud, promueve flujos de informaciones acerca de las dolencias corporales, contextos familiares y de vida, que extienden y diversifican los modos de escuchar sobre los casos, más allá de los límites de la consulta.

\section{Los márgenes de la escucha o escuchar en los márgenes}

Cuando Patricia narraba el "caso de Rosario", me describió en detalle la estafa que esta paciente había sufrido por parte de un programa de viviendas de una ONG "solidaria", que se dedicaba a solidarizar lo ajeno como propio. Frente a una mezcla entre angustia y bronca por la pérdida de sus ahorros, diferentes personas del Centro, incluso Patricia, ayudaron a Rosario a resolver el problema, es decir, no sólo a realizar las denuncias, sino a recuperar su dinero, y ahora poder proyectar una vivienda real.

Esto lo llamo desamparo profundo. Por ahí las condiciones de empleo es... precarizada o de desempleo total... Analfabetismo; la falta de escolarización o la deserción escolar...; las situaciones habitacionales indignas... Me parece que es algo que caracteriza al barrio y que da como una... ¿ ¿No?, como que le da cierto... y esta perspectiva de que algo del padecimiento no es sin el contexto histórico del malestar, me parece que... que es algo que... era como un obvio... que mi formación de grado y de la residencia... No estaba en mi formación. O estaba pero como una obviedad... y acá se pone... Digamos, yo siento que se pone en práctica. Y que si no lo considero... No sé... no se puede... (Patricia, psicóloga).

Examinando este y otros casos, los psicólogos cuestionaban los contenidos y la relación entre lo obvio y lo excepcional respecto del repertorio de dolencias y malestares en contextos de pobreza urbana. Frente a este problema, lo obvio y 

márgenes, que se resistían a las condiciones de legibilidad e inteligibilidad, a los códigos de lectura, de categorización y de trabajo con la palabra. Mientras que las matrices de saberes y técnicas expertas psi daban las condiciones de articulación entre lo audible y lo inteligible "obvia" y "tradicional" en el despliegue terapéutico, otros repertorios de saberes y técnicas, de modos de sentir y escuchar, van dando forma a las tecnologías terapéuticas.

A diferencia de las categorías diagnósticas psi que atraviesan cualquier práctica en diferentes contextos sociales, los psicólogos generalmente expresaban en términos de problemas y malestares que combinan lenguajes expertos y legos : "desamparo", "vulnerabilidad”, "desempleo”, "peligrosidad”, "pánico”, "violencia”, "abuso", "crimen”, "trata”, etc.

La gente que viene con historia de abuso, pobreza, desempleo... Todas esas cosas así, que las escuchás a diario..., las escucho, es muy difícil... porque hay un punto donde vos no podés obrar sobre eso, no tenés la barita mágica para solucionar eso..., pero yo lo puedo como... lo puedo escuchar, lo puedo recibir. Y... sostener esa angustia, ese malestar. Mi pertenencia a la misma clase social creo que me ayuda en ese sentido... (Clara, psicóloga).

Como Clara expresa, este lenguaje define cartografías teóricas-empíricas que refieren a esos otros que viven otras realidades. Estas experiencias y realidades son sentidas, vividas, objetivadas, escuchadas y expresadas por los profesionales no sólo de acuerdo a las matrices de inteligibilidad psicoanalítica, en la que se han formado. En la formación y desarrollo de la prácticas, otros saberes, técnicas y fuentes de inteligibilidad se van agregando: otros saberes expertos (ej. salud colectiva, ciencias sociales, etc.), modos de sentir, entender, hablar y escuchar propias de los sectores populares, perspectivas y categorías de poblaciones marginalizadas que pertenecen a estos conjuntos sociales. De acuerdo a los propios profesionales, la capacidad de sentir en general, y de escuchar en particular, está en relación también con los años de experiencia en tratamientos, la desigualdad y distancias sociales y territoriales de las experiencias vividas, y a la diversidad y flexibilidad del repertorio de modos de decir, escuchar y atender.

Después, no sé, creo que con los años también... eh... no sé si... cada vez es menos lo que no podés escuchar, capaz... Como que te vas haciendo medio callo... Porque vas escuchando... no escuchás la anécdota en sí misma... Porque creo que ahí está la diferencia entre un analista y un amigo... o alguien que se conmueva con lo que vos les estás diciendo, sino que tenés que escuchar otras cosas, que eso me parece que... está 
bueno para organizar que no te desborde cada vez que escuchás a esta gente que está

sufriendo. Que es escuchar... otra cosa de eso que dice... En ese padecer que es un espanto. A veces hay cosas son espantosas, son una mierda que no podés concebir... y ahí tenés que correr y pensar... (Laura, psicóloga).

Como Laura refiere, en estas poblaciones, las dificultades en la escucha en los tratamientos, se pueden formular en términos de las relaciones entre lo inteligible y lo audible, es decir, refiere al mapa que diferencia y articula entre sonidos, sentidos, silencios y ruidos. También, estas relaciones entre lo audible e inteligible se desarrollan y despliegan en diversas temporalidades: institucionales, terapéuticas, de la vida cotidiana. Las tensiones y conflictos que resultan de esta diversidad de temporalidades, además, imponen a la escucha experta exigencias de categorización temporal de diferente orden ("lo urgente", "el riesgo", "la emergencia" y el "peligro"), y que, en ocasiones, desencadenan diferentes secuencias de acción e intervención más allá de la consulta.

Estos saberes y técnicas expertas y legas, sin embargo, definen las condiciones de legitimidad y reconocimiento de los malestares que los pacientes expresan. Por lo tanto, la pregunta sobre la subjetividad se convierte en secundaria y sólo puede ser formulada como una derivación, un resultado de la calibración de esta compleja matriz entre lo inteligible, audible que atraviesan los contextos terapéuticos en realidades sociales otras. Como Patricia refiere, escuchar ciertas experiencias y realidades ya no sólo conmueve las nociones de sujeto y persona dominantes en los saberes expertos psi. También, desencadena cuestiones morales que interrogan acerca de un cierta particular otredad, producida por el extrañamiento de otras y extremas experiencias de vida: “¿cómo alguien puede vivir eso y tener una vida?", "te quedás asi preguntando: tengo que escuchar o tengo que intervenir por fuera?" "te lleva tiempo ver que cosas asi le pasen a la gente? "necesito hablarlo con otros, es mucho para mi”.

En aquellos casos, como el de Rosario, que se convierten en paradigmáticos en los Centros de Salud, lo que se escucha excede, subvierte e interpela el repertorio de problemas modelado durante el proceso de formación profesional. Es decir, los vínculos esperados entre lo decible, audible y en el contexto terapéutico se ven profundamente conmovidos. La ruptura de las coordenadas tradicionales respecto de los modos que asumen los malestares y sufrimientos en contextos de pobreza urbana, no sólo marca a la escucha experta psicoanalítica como algo insuficiente. Por un lado, promueve un proceso de expertización, es decir, 
una tendencia a reducir y subsumir dentro las matrices de saberes y técnicas expertos los diferentes malestares, problemas y dificultades de los pacientes y de las poblaciones. Por otro lado, promueve un proceso de popularización, en el cuál se amplía el registro de los sensible, incluyendo modos de hablar, escuchar, ver, atender y entender (modismos y expresiones locales sobre los malestares y emociones, tipos y modos de habitar los territorios y viviendas, ritmos cotidianos, y modelos de sociabilidad, etc.) provenientes a los sectores populares que se atienden y, en algunos pocos casos, al que también pertenecen los profesionales. Por último, un proceso de marginalización, por el que se extiende y diversifica el registro de lo sensible, con la incorporación de matrices de inteligibilidad, modos de sentir (escuchar, ver, etc.) y decir, que pertenecen a sectores marginalizados que viven en barrios populares. Con esta expresión se incluyen problemas, malestares y consecuencias de diversas economías ilegales (drogas, prostitución, trata, etc.), sus modos de sujeción particulares, peligros y consecuencias vinculados también con las fuerzas represivas de las mismas.

De la conjunción de estos procesos, no sólo se amplían las coordenadas expertas de lo sensible, audible e inteligible, sino que también se reconocen y en ocasiones, incluyen otras matrices de inteligibilidad legas, populares y marginales, las que ampliando y flexibilizando los modos de sentir, en algunas ocasiones, se convierten en receptivas, es decir, sensibles respecto de los modos de vida de estas poblaciones. Sin embargo, el desarrollo de estos procesos que tienen características, contenidos y orientaciones contrapuestas, produce también nuevas tensiones, conflictos y experiencias de malestar incluso para los propios profesionales. Los psicólogos expresaban múltiples efectos y consecuencias de la confrontación con otros que viven otras realidades en contextos de pobreza urbana. Entre los diferentes efectos, encontramos los siguientes: dificultades para dormir e incluso insomnios prolongados; temores, miedos e incluso pánico por peligros en los pacientes y consecuencias indirectas para los propios profesionales; accidentes frecuentes con heridas y fracturas; contracturas crónicas; excesos en el consumo de sustancias; específicamente alcohol y psicofármacos; cambio de lugar o incluso renuncia al trabajo.

En la confrontación con otros que viven otras realidades, lo que queda por fuera de los registros de lo sensible definido por las matrices de los saberes y técnicas expertas, se articulan con aquellos otros saberes y prácticas de los 
sectores populares y de las poblaciones marginalizadas, y se convierten ya sea en ruido o sonidos sin sentido, en sonidos que se reducen e integran a sentidos ya conocidos, o con sentidos otros, desconocidos en espera y en desarrollo. Es decir, en este despliegue el repertorio de modos de sentir (corporales, emocionales y perceptuales), se incluyen saberes legos, populares y marginales, no sólo en los tratamientos y experiencias en Centro de Salud, sino también en las intervenciones barriales. Basada en estas perspectivas, se hace posible sistematizar, aunque sea de forma aproximada, diferentes modalidades de escucha psicológica dentro de un conjunto heterogéneo.

Algunos pocos psicólogos, más precisamente dos, tendían a reducir y capturar la diversidad de las experiencias y realidades a las categorías psi tradicionales que colectan la amplia variedad de problemas vinculados con la pobreza urbana. Cuando esta escucha restrictiva y reductora, que transforma y traduce lo desconocido a lo conocido, se convierte en insuficiente, el caso o bien era derivado a otros psicólogos, o bien los pacientes dejaban el tratamiento. La mayoría de los psicólogos, sin embargo, buscan ampliar y modificar, diversificar y ampliar los registros de lo sensible y lo inteligible. El proceso de extender la escucha más allá de la experticia tradicional, se realiza a través de diversos procesos institucionales: análisis grupal de los casos, formación teórica y técnica en psicoanálisis, ateneos, supervisiones, el psicoanálisis personal, e intervenciones en las mismas viviendas de los pacientes por parte de miembros del equipo de salud. También, se incluye la formación en otras orientaciones y disciplinas que problematizan de modo diferente los malestares y dolencias de la pobreza urbana, (como por ejemplo la salud colectiva). No obstante, este tipo particular de articulación entre lo audible y lo inteligible, produce lo que podríamos denominar una escucha fragmentada, es decir, una escucha que multiplica y diversifica las acciones de oír, ver y hablar, más allá de los bordes de la experticia psi, incluyendo diversos interlocutores profesionales y legos, y diferentes secuencias de acción e intervención. Sin embargo, la multiplicación de los flujos de información y de referencias sobre los pacientes dentro de la institución, complejizan y, en ocasiones, imposibilita integrarlos en una figura tradicional del caso, ya que involucra el desarrollo y/o articulación con otros tipos de saberes, tecnologías e intervenciones expertas (trabajo social, judicial, programas de vivienda, etc.) y legas (líderes y organizaciones comunitarios, movimientos de lucha, partidos políticos, etc.). 
Para otros -también pocos- psicólogos, finalmente, la confrontación con estas poblaciones, ha promovido la producción y revisión de las matrices expertas, del psicoanálisis, de las técnicas analíticas, de los vínculos entre realidades psíquicas y sociales, y de los modos de abordar a través de políticas específicas, diferentes "sufrimientos sociales" contemporáneos. Esta escucha en formación continua, se convierte en una escucha informada de las transformaciones de las realidades sociales y de los malestares que emergen de ellos, también incluye críticas de otras disciplinas, como la revisión de la psicologización de la pobreza a través de las psicoterapias, de las diferentes psicopatologías y criterios diagnósticos, y las críticas de los encierros como estrategia terapéutica.

\section{Palabras finales}

Cuando en nuestro primer encuentro Patricia comenzó a describir "el caso Rosario", algo en el modo de decir sus detalles, hacía audible en las variaciones de tonos y sus intensidades, sus silencios y énfasis, ciertas particularidades del mismo. Elevando el tono en un in crescendo que expresaba el nivel de "horror", Patricia hacía claramente audible al hablar, lo que le había producido la escucha de relatos de Rosario acerca de sus experiencias de maltrato y abuso. Patricia, como otros psicólogos en éste o en otros casos, intentaban capturar con diversas expresiones, los modos de sentir y entender la escucha en estos tratamientos: "exceso", "demasiado", "es mucho para todos los días", "no era fácil de escuchar", "no era creíble", "un espanto", "era una novela... de terror".

Estas dificultades y particularidades de la tecnología experta de escuchar se hacen claramente evidentes en aquellos casos paradigmáticos, aunque atraviesan y modelan los tratamientos centrados en la palabra en los Centros de Salud barriales. Por un lado, viene a mostrar la insuficiencia y a cuestionar la eficiencia de las matrices psi que articulan y dan forma a la escucha experta tradicional cuando es atravesada por los cambiantes patrones de la desigualdad social y de segregación territorial, la diversificación de los saberes y prácticas psi, de las lógicas de violencia institucional, barrial y de género, la modificación de las características y reducción de las redes de sociabilidad locales. Por otro lado, revuelven, cuestionan y obligan a reformular ciertas dicotomías que sustentan las técnicas expertas de escuchar: teorías y técnicas, lo ordinario y extraordinario, la realidad psíquica y social, lo universal y lo contextual, lo experto y lo lego, 
el adentro y afuera - subjetivo e institucional -, el secreto y lo público, la intervención terapéutica y social, las realidades vividas y las otras realidades.

La reflexividad psicológica tanto sobre estos excesos y dificultades, como sobre los fundamentos y matrices que los fundamentan, han producido la inclusión de otros modos de escuchar, hablar y ver, han modificado los registros de lo sensible, las articulaciones entre lo decible, audible e inteligible, expresadas en diferentes modalidades de escucha: reductiva, fragmentada y en formación. Esta reflexividad incluye, a su vez, una interrogación constante y, a veces, angustiante, sobre los alcances, posibles efectos y eficacia de los tratamientos. Sin embrago, y al ritmo de estas interrogaciones sobre la propia actividad, los psicólogos refieren una multiplicidad de efectos y eficacias terapéuticas: alivio, revelación de secretos dolorosos, sentirse acompañado en condiciones de soledad y aislamiento, abrir los dolores producidos por diferentes pérdidas, dejar de (auto) medicarse con psicotrópicos, poder salir de cotidianeidades violentas, suspender los suicidios, trazar nuevas formas de sociabilidad, etc.

Finalmente, y ubicando la descripción y análisis de la tecnología de la escucha experta psicológica en el marco de un estudio etnográfico y barrial más amplio, se hace posible localizar y articular los procesos de modificación de las tecnologías psi (expertización, popularización y marginalización) en un contexto de rápida transformaciones sociales en contextos de pobreza urbana. Por último, las tensiones dentro del dominio de las tecnologías terapéuticas, entre tratamientos centrados en la palabra y poblaciones a las que están dirigidas, entre sistemas públicos de salud y complejas realidades sociales, participan, son producidas, y en algunos casos subvierten, los modos de gobierno de lo sensible, de la pobreza y de la marginación en estos contextos urbanos.

\section{Referencias}

BARTHES, R. Lo obvio y lo obtuso. Imágenes, gestos y voces. Barcelona: Paidós, 1986. 382 p. BIEHL, J.; GOOD, B.; KLEINMAN, A. (Eds.). Subjectivity. Ethnographic investigations. Berkeley: University of California Press, 2007. 477 p.

DAGFAL, A. Entre París y Buenos Aires. La invención del psicólogo (1942-1966). Buenos Aires: Paidós, 2009. 578 p.

DAMOUSI, J.; PLOTKIN, M. The Transnational Unconscious. Essays in the History of Psychoanalysis and Transnationalism. New York: Palgrave Mc Millan, 2009. 256 p. 
DUARTE, L. F. D. Da vida nervosa nas classes trabalhadoras urbanas. Rio de Janeiro: Zahar, 1986. $290 \mathrm{p}$.

EPELE, M. El tratamiento como palimpsesto. Cuando la medicalización se convierte en crítica políticamente correcta. Cuadernos de Antropología Social, v. 38, p. 7-31, 2013.

- Etnografía, fragmentación social y drogas: hacia una política de las miradas.

Etnografías contemporáneas, v. 3, n. 3, p. 117-143, 2007.

. Sujetar por la herida. Buenos Aires: Paidós, 2010. 298 p.

ESCUDERO, J. The health crisis in Argentina. International Journal of Health Services, v. 33, n. 1, p. 129-136, 2003.

FASSIN, D. Exclusion, underclass and marginality. Figures contemporaines de la pauvrete urbaine en France, aux ÉtatsUnis et en Amérique Latine. Revue Francaise de Sociologie, v. 37, n. 1, p. 37-75, Jan-Mar 1996.

FERNÁNDEZ, A. Crítica y clinica. Desafíos psicoanaliticos frente a subjetividades actuales. Revista Subjetividades. Psicoanálisis de las configuraciones vinculares, tomo XXXVI, n. 1-2, 2013.

FOUCAULT, M. El gobierno de si y de los otros. Curso del College de France, Buenos Aires: Fondo de Cultura Económica, 2009.

FREUD, S. Consejos al médico en el tratamiento psicoanalítico [1912]. In: . Obras Completas, Tomo XII, Buenos Aires: Amorrortu, 1979a. 424 p. . Nuevos Caminos de la Terapia Analítica [1918]. In: . Obras Completas,

Tomo XVII. Buenos Aires: Amorrortu, 1979b. 320 p.

INGOLD, T. The perception of the environment. Essays on livelihood, dwelling and skill. London: Routdlege, 2000.

IRIART C.; WAITZKIN, H. Argentina: no lesson learned. International Journal of Health Services, v. 36, n. 1, p. 177-196, 2006.

JAY, M. Downcast eyes: the denigration of vision in twentieth-century French thought. Berkeley: University of California Press, 1993.

LACAN, J. Escritos 1. Buenos Aires: SXXI, 2008.

LAKOFF, A. The Pharmaceutical Reason. Cambridge: Cambridge University Press, 2005. LOCK, M.; NGUYEN, K. An anthropology of biomedicine. Oxford: Wiley-Blackwell, 2010. MERLEAU-PONTY, M. Fenomenología de la Percepción. Buenos Aires: Planeta Agostini, 1986.

ONOCKO-CAMPOS, R. et al. Salud Colectiva y Psicoanálisis: entrecruzando conceptos en busca de políticas públicas potentes. Salud Colectiva, n. 4, p. 172-185, 2008. 
ORTIZ-HERNÁNDEZ, L.; LÓPEZ MORENO, L.; BORGES, G. Desigualdad económica y salud mental: revisión de la literatura latinoamericana. Cad. Saúde Publica, v. 23, n. 6, p. 1255-1272, 2007.

PLOTKIN, M. Freud en las Pampas. Buenos Aires: Editorial Sudamericana, 2003.

ROSE, N. Inventing ourselves. Psychology, Power and Personhood. Cambridge: Cambridge University Press, 1998.

STOLKINER, A. ¿Qué es escuchar a un niño? Escucha y hospitalidad en el cuidado de la salud. In: DUEÑAS, G.; KAHANSKY, E.; SILVER, R. (Eds.). La patologización de la infancia III. Buenos Aires: Noveduc, 2013. p 71-84.

STOLLER, P. The taste of ethnographic things: the senses in anthropology. Philadelphia: University of Pennsylvania Press, 1989.

SVAMPA, M. La Sociedad excluyente. La Argentina bajo el signo del Neoliberalismo. Buenos Aires: Taurus, 2005.

VAN EDE, Y. Sensous anthropology: sense and sensibility and the rehabilitation of skill. Anthropological Notebooks, v. 15, n. 2, p. 61-75, 2009.

VEZZETTI, H. Freud en Buenos Aires. Buenos Aires: Universidad Nacional de Quilmes, 1996.

. La Locura en Argentina. Buenos Aires: Paidós, 1985.

VISACOVSKY, S. ElLanús. Memoria y política en la construcción de una tradición psiquiátrica y psicoanalítica argentina. Buenos Aires: Alianza Editorial, 2002.

. La Constitución de un sentido práctico. Del malestar cotidiano y el lugar del psicoanálisis en la Argentina. Cuicuilco, v. 45, p. 51-79, 2009.

\section{Notas}

${ }^{1}$ Desde esta perspectiva se ha edificado la oposición ya tradicional entre la visión que distancia, objetiva, atomiza y analiza, y se convierte en el centro sensorial de la producción del conocimiento y del acceso a la verdad, por un lado, y el oír y escuchar subjetiva, integra, penetra en el interior y participa en la modulación de los sentimientos (MERLEAU PONTY, 1986; INGOLD, 2000).

${ }^{2}$ Desde los primeros desarrollos del psicoanálisis con Freud, el acto de escuchar, como opuesto al oír, ha perdido su naturalidad y transparencia. En diferentes momentos, Freud detalla y revisa las particularidades que convierten al escuchar, una técnica analítica, la atención flotante, que combina de forma inédita la atención con la evitación de la crítica selectiva, agendas prefijadas y expectativas teóricas (FREUD, 1979a; 1979b). En consejos al médico sobre el tratamiento psicoanalítico, Freud detalla ciertas características que hacen del escuchar, una técnica analítica, "la escucha". Bajo el nombre de la atención flotante, amalgama dos componentes en apariencia irreconciliables en la escucha analítica, la atención por un lado, y la evitación de su fijación sólo en aquellos fragmentos que las expectativas e inclinaciones del analista pudieran seleccionar voluntaria y conscientemente, 
por el otro. Con posterioridad, fue precisando las variaciones técnicas de acuerdo a diferentes padecimientos. Específicamente, en el contexto de posguerra, Freud abre la posibilidad de extender el psicoanálisis a las clases populares, con tratamientos estatales y gratuitos, en los cuáles las técnicas deberían ser modificadas respecto a las particularidades de estas poblaciones (FREUD, 1979b). En desarrollos posteriores del psicoanálisis, la escucha ha sido revisada y revisitada de acuerdo a las diferentes orientaciones teóricas y técnicas. Cuestionando el modelo estratigráfico que hace corresponder diferentes profundidades con la topología del aparato psíquico, las continuas revisiones desde Lacan sobre la escucha trabaja en la compleja tensión entre el decir, lo dicho, y los silencios. Lejos de buscar asimilar el sentido de lo dicho a ciertos contenidos teóricos, la escucha atiende allí dónde las palabras se quiebran, tambalean, hacen silencio, se callan, no se puede decir, o se dice una cosa por otra, entre las principales (LACAN, 2008).

${ }^{3}$ Desarrollos recientes, sin embargo, han incluido al problema de la escucha a la luz de la noción de hospitalidad, de alojar al otro (STOLKINER, 2013). De acuerdo a Stolkiner, la escucha supone tanto un acto de subjetivación como una tendencia a la objetivación a través de los conocimientos y diagnósticos. Siguiendo a Derrida, para esta autora, alojar, entonces, remite a este acto que supone el amparar al otro, en su extranjería, en su otredad. 
Between listening and hearing:

psychoanalysis, psychotherapy and urban poverty in Buenos Aires

Psychoanalysis in Argentina has been studied by different approaches and Social Sciences; however, only a few studies have investigated it within the public health system, specifically in hospitals. Based on the results of the ethnographic research carried out in Health Centers in a neighborhood of the Buenos Aires Metropolitan Area, this paper aimed to problematize the listening technique, specifically, the modes of listening that participate in those treatments based on speech and orientated to marginalized populations. By articulating the anthropological perspectives on health, psychotherapies and psy technologies on the one hand, and on the sense on the other, in this paper the modes of listening are understood and analyzed as perceptual, corporal and expressive actions. Finally, based on a ethnographic approach, it is suggested that to hear and to listen, being listened and to speak within the treatments, can be understood as only one case related to a complex set that these actions are carried out in everyday life.

> Key words: psychoanalysis; psychotherapy; urban poverty; ethnography; Buenos Aires. 
Entre a escuta e o escutar: psicanálise, psicoterapia e pobreza urbana em Buenos Aires A partir de diferentes disciplinas e perspectivas tem se pesquisado as diversas técnicas, orientações e desenvolvimentos da psicanálise na Argentina; no entanto, somente alguns poucos estudos se dedicaram a pesquisar as psicoterapias e a psicanálise no sistema público de saúde, especificamente em hospitais. Partindo dos resultados da etnografia que vem sendo realizada desde 2013 em Centros de Saúde em um bairro da Área Metropolitana de Buenos Aires, este trabalho tem por objetivo problematizar a escuta. Isto é, os modos de escutar em sua diversidade que participam como tecnologias nos tratamentos centrados na palavra e orientados às populações marginalizadas. Através da articulação das perspectivas antropológicas que abordam as psicoterapias e tecnologias $p$ si em populações marginalizadas e aquelas que estudam os sentidos, serão analisados aqui os modos de escutar em termos de ações corporais, de modo perceptivo e expressivo. Finalmente, e baseada em uma aproximação etnográfica da comunidade/bairro, o ouvir e o escutar, o ser escutado e o falar nos tratamentos centrados na palavra, se convertem em uma variação entre os diversos modos que estas ações assumem na vida cotidiana destas populações.

> Palavras-chave: psicanálise; psicoterapia; pobreza urbana; etnografia; Buenos Aires. 